\title{
The Evolution of Compact Radio Sources
}

\author{
Paul Alexander* \\ Cavendish Laboratory, JJ Thomson Avenue, Cmbridge, CB20HE, UK \\ E-mail: pa@mrao.cam.ac.uk
}

\begin{abstract}
Once considered exotic, or even esoteric, Active Galactic Nuclei (AGN) and radio sources have in recent years gained a central role in attempts to understand galaxy evolution. Theoretical models of galaxy evolution invoke either "quasar-mode" or "radio-mode" feedback, or some combination, from energetic AGN. The aim is to solve problems with the standard hierarchical galaxy formation scenario especially at the high-mass end of the galaxy mass function. However the details and likely efficiency of this feedback process are not well understood and therefore both observational and theoretical studies to investigate these issues are at the present time very topical. In particular, to understand radio-mode feedback we need to understand the physics and evolution of radio sources on galactic scales - i.e. compact radio sources. Here I present an overview of model which describes the evolution of radio sources from the smallest scales (of order parsecs) through to the well-established near self-similar evolution on scales of 10's to 100's of kiloparsecs. The aim of the model is to elucidate some of the key physics which is needed to understand radio source evolution on galactic scales and how hence the efficiency and nature of radio-mode feedback. Other important aspects of the source evolution, such as jet-stability, the effects of finite external pressure on the dynamical evolution, will be discussed elsewhere.

Richard Schilizzi, through his pioneering work in Very Long Baseline Interferometry (VLBI) has made some of the most important contribution to the study of compact radio sources. As we move into the era of the Square Kilometre Array (SKA) there is a very exciting opportunity to observe in detail radio-mode feedback in operation.
\end{abstract}

Resolving the Sky - Radio Interferometry: Past, Present and Future -RTS2012

April 17-20, 2012

Manchester, $U K$

${ }^{*}$ Speaker. 


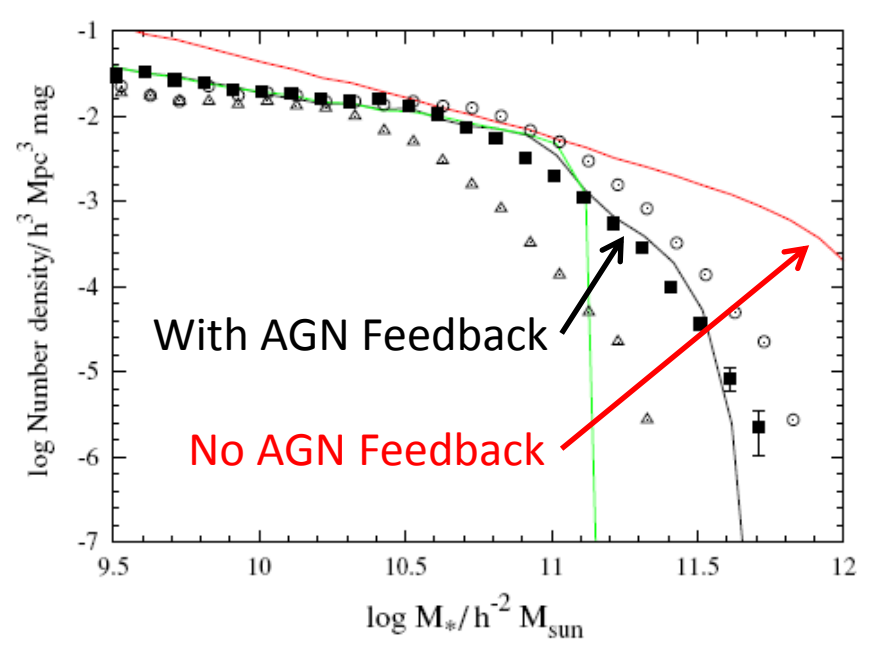

Figure 1: Effect of incorporating radio-mode feedback in galaxy evolution models from [1]

\section{Introduction}

It is now well-established observationally [2] and theoretically [3] that radio-loud Active Galactic Nuclei (AGN) have a significant impact on their environment on large scales for example providing heating [4],[5] and driving dynamical processes in clusters [7],[?]. More recently the possibly crtical role played by AGN, and and again radio-loud AGN, in galaxy evolution has become a matter of intense interest. It is clear that the energy output of AGN is very significant when considered alongside other possible sources of energy providing feedback during galaxy evolution (See Figure 1), however the physical mechanisms by which this energy output can be efficiently coupled to the host galaxy's Interstellar Medium (ISM) is not well understood. Commonly two possible modes are considered: the Quasar mode where the energy output is in the form of a photon flux, or the radio-mode in which the AGN produces jets and hence a radio source. Radio-mode potentially provide interesting ways of coupliing the AGN output to the ISM via fluidynamical proceses which can be efficient at both heating gas, but also potentially moving gas to beyond the cooling radius.

For radio-mode feedback in galaxies we are are clearly concerned with radio sources smaller than the physical extent of the galaxy which means we need to understand the physics and especially evolution of compact radio sources. In this paper I will present a dyamical model for the evolution of such radio sources which is physically likked to the sucessful self-similar model for radio sources we developed some years ago [8], and therefore provides self-consistent decription of the evolution of the radio source out to the large physical scales.

\section{Characterist Length-Scales and Self-Similar Evolution}

On the largest scales, which I will quantify shortly, the evolution of radio sources is well described as self-similar or scale-invariant. This is illustrated in Figure 2 in which I show five 


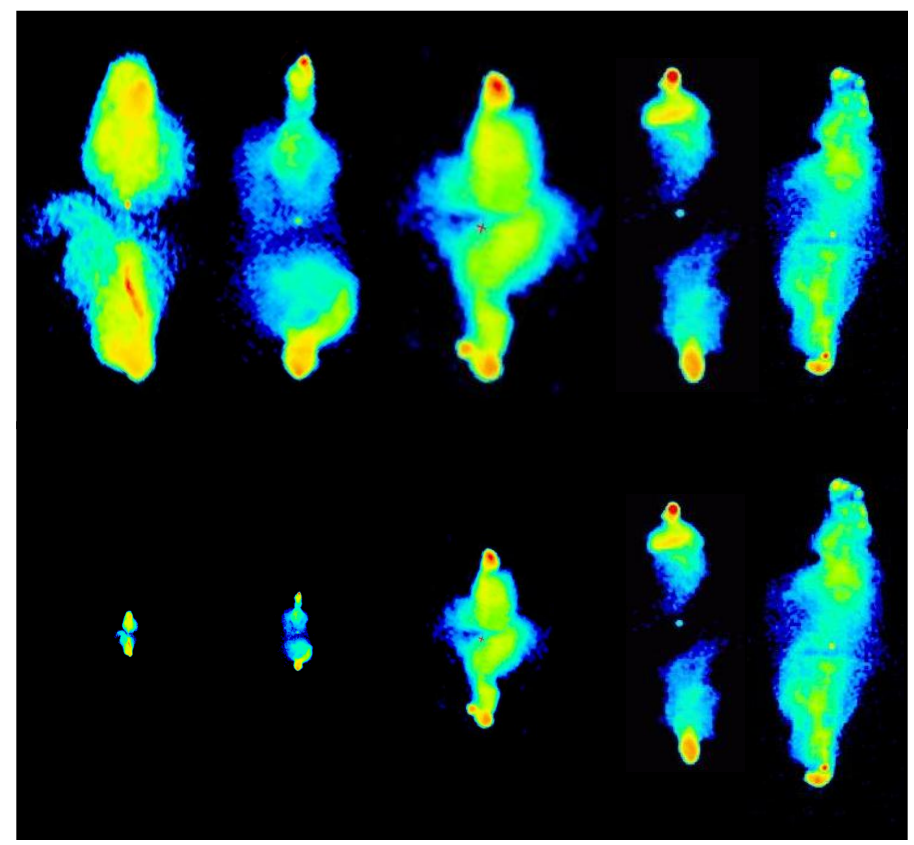

Figure 2: Illustrating the self-similar evolution of radio sources. Upper panel shows five radio source images all to the same size while the lower panel shows the images scaled to the sources true linear size.

examples of real radio sources in two ways, firstly all scaled to the same size and secondly scaled to their actual physical size. By examining the top panel it is not possible to deduce the physical size from the observed structure - in many ways this is a great dispaointment since if the structure were dircely related to physical size then radio sources would have provided us with a superb standard rod, but unfortunately nature deosn't work that way!

It is possible to construct a physical model to decribe this situation. We consider a jet which is initially uncollimated and characterise the radio source just by the properties of the jet which we take to be the mass flow rate $\dot{M}=A_{j} \rho_{j} v_{j}$, the jet kinetic power $Q=\frac{1}{2} A_{j} \rho_{j} v_{j}^{3}$ and the solid angle of the uncollimated jet $\Omega$. The final dimensional quantity we require is the external density $\rho_{X}{ }^{1}$. The external pressure is not important provided the momentum flux of the jet greatly exceeds the external pressure and the cocoon which must form around the jet is highly over-pressured with respect to the external environment. $A_{j}, v_{j}$ and $\rho_{j}$ are the jet cross-sectional area, density and velocity the corss-sectional area and density can vary along the jet, however constancy of both the jet mass flux and kinetic power require the jet velocity to be constant.

From this set of dimensional quantities we can form two related length scales :

$$
L_{1}=s \sqrt{2}\left(\frac{Q}{\rho_{X} v_{j}^{3}}\right)^{1 / 2}=56\left(\frac{Q}{10^{39} \mathrm{~W}}\right)^{1 / 2}\left(\frac{\rho_{X}}{10^{-22} \mathrm{~kg} \mathrm{~m}^{-3}}\right)^{-1 / 2}\left(\frac{v_{j}}{c}\right)^{-3 / 2} \mathrm{pc}
$$

and

$$
L_{1 b}=\frac{1}{2 \Omega^{1 / 2}} L_{1}
$$

\footnotetext{
${ }^{1}$ The analysis remains straightforward even when the external density is varying in which case a characteristic external density must be specified.
} 


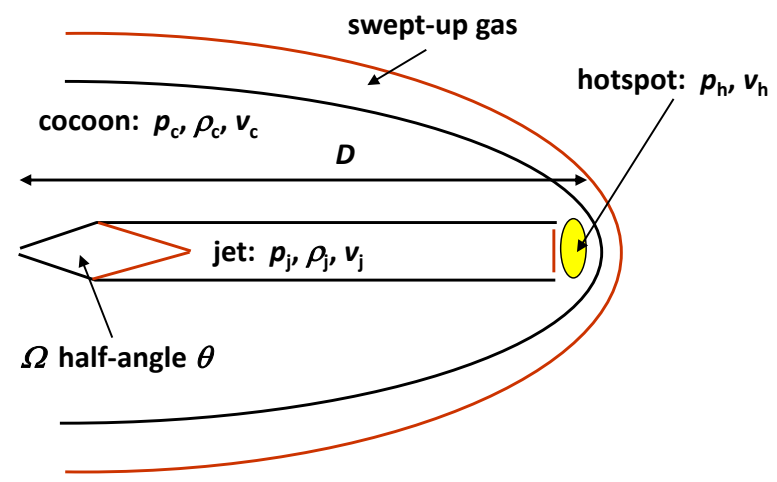

Figure 3: Figure illustrating the main components of the self-similar radio source model

To understand their physical significance I write $L_{1}$ in the following form, noting that for the conical flow of the uncollimated jet the density decreases with distance from the origin, $D$ as $1 / D^{2}$ while the cross-sectional area increases as $D^{2}$

$$
L_{1}^{2}=8 \frac{Q}{\rho_{X} v_{j}^{3}}=4 \frac{A_{j} \rho_{j} v_{j}^{3}}{\rho_{X} v_{j}^{3}}=4 \Omega D^{2} \frac{\rho_{j}}{\rho_{X}}
$$

Since $\rho_{j}$ is a decreasing function of $D$ we deduce that $L_{1 b}$ is the distance from the origin when the jet density becomes equal to the external density, and for larger scales the jet is always lighter than the external environment. For source size $D>>L_{1 b}$ we expect the flow to become independent of $L_{1 b}$ and since there is no other characteristic length scale we therefore expect a self-similar solution to exist for the source evolution. Simple dimensional arguments lead to a dependence of the source size on time of $D \propto\left(Q t^{3} / \rho_{0} a_{0}^{\beta}\right)^{1 /(5-\beta)}$ where this is generalised for the case of an atmosphere in which $\rho_{X}=\rho_{0}\left(r / a_{0}\right)^{-\beta}$.

Kaiser and Alexander [8] provide a detailed analysis of this phase, but we can understand the physical origin of the self-similar solution straightforwardly. For the conical flow, since the jet density declines with distance so too does the momentum flux. The sideways momentum flux of the jet is lower than the forward momentum flux by a factor of $\sin ^{2} \theta \sim \Omega$ where $\theta$ is the half-angle of the uncollimated jet which I take to be small. Assuming that a cocoon exists, then at some point along the jet length the side-ways momentum flux becomes equal to the cocoon pressure (see Figure 3). The cocoon pressure then collimates the jet by driving an oblique shock into the jet. The jet, which we can initially assume was cold now acquires a pressure $p_{j} \sim \theta^{2} \rho_{j} v_{j}^{2}=p_{c}$ which is equal to the cocoon pressure. If the sound speed in the cocoon is high the cocoon pressure has no spatial gradient (although is does evolve with time) and the jet forms a well-collimated flow all the way to the hotspot. Since $\rho_{j} v_{j}^{2}$ is the forward momentum thrust and $p_{c}$ drives the sideways expansion the fact they remain in proportion to one another gives the self-similar behaviour.

Elegant demonstration that this form of solution applies to shown by the observations of de Vries et al. [9] for which RTS has been a long-term collaborator (Figure 4). Here the direct expansion velocity observed by VLBI is shown to follow the expected time dependence of the model just outlined. 

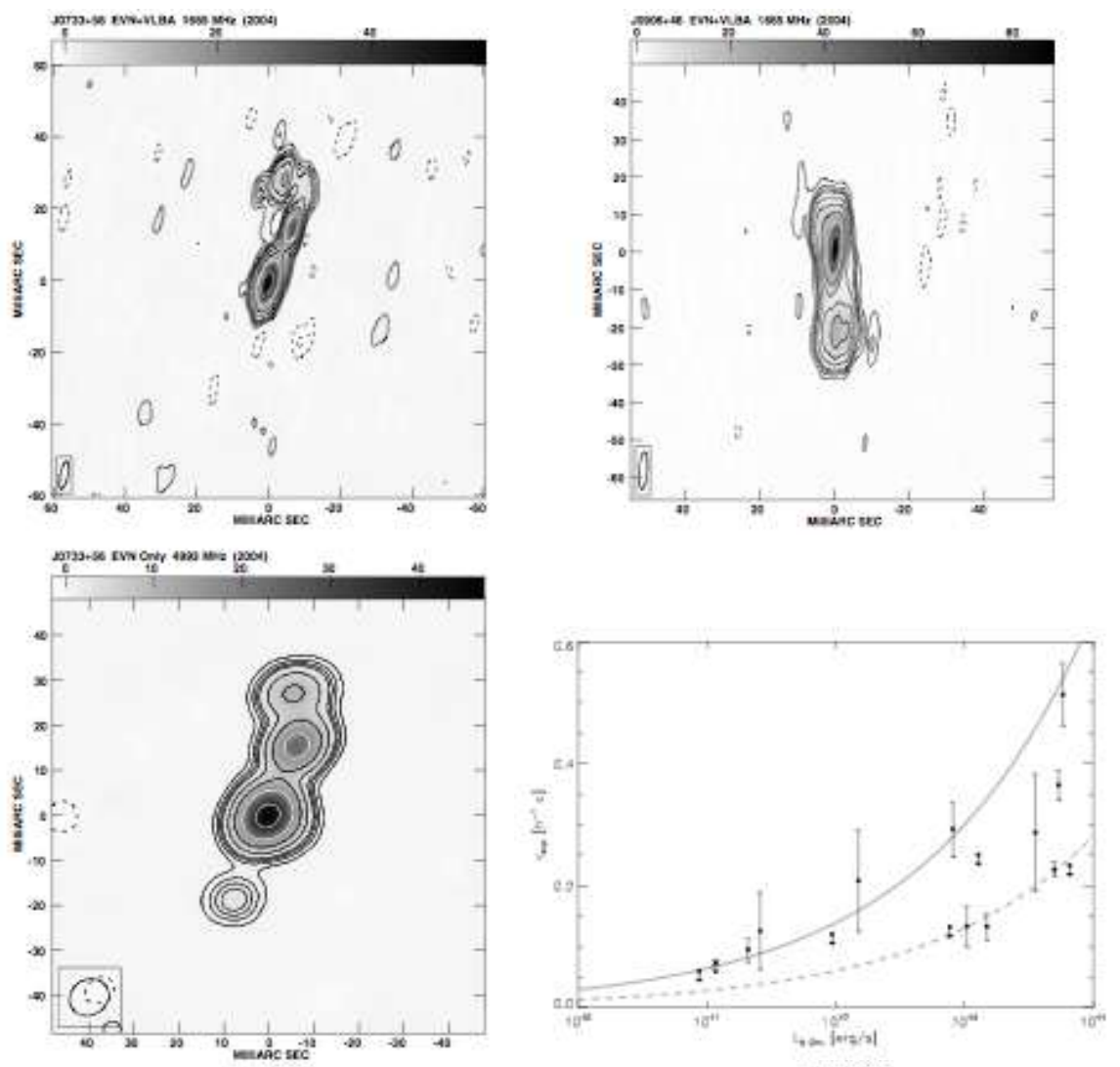

Figure 4: VLBI observations show direct evidence for self-similar evolution [9]

\section{Early Evolution to Re-collimation}

To extend the model I have just outlined I consider two regimes. Firstly to develop a model for the evolution of the radio source for scale sizes less than size on which the jet is first collimated and secondly to consider jet collimation itself. The jet is modelled as a cononical uncolimated flow characterised in exactly the same way as in the self-similar case by the jet mass flux, kinetic energy flux and the solid-angle of the jet: again the external density is key parameter and for the small physical scales now relevant I consider only a constant external density. Further assumptions are needed

- The expansion is highly supersonic hence we can use strong-shock approximations and more importantly can assume the bow-shock surrounding the source is always close to the contact surface.

- The sound speed is sufficiently high that pressure variations in the cocoon can be neglected.

- The hotspot can be treated as a seperate dynamical region in with different pressure and volume through which jet material flows to the cocoon. 

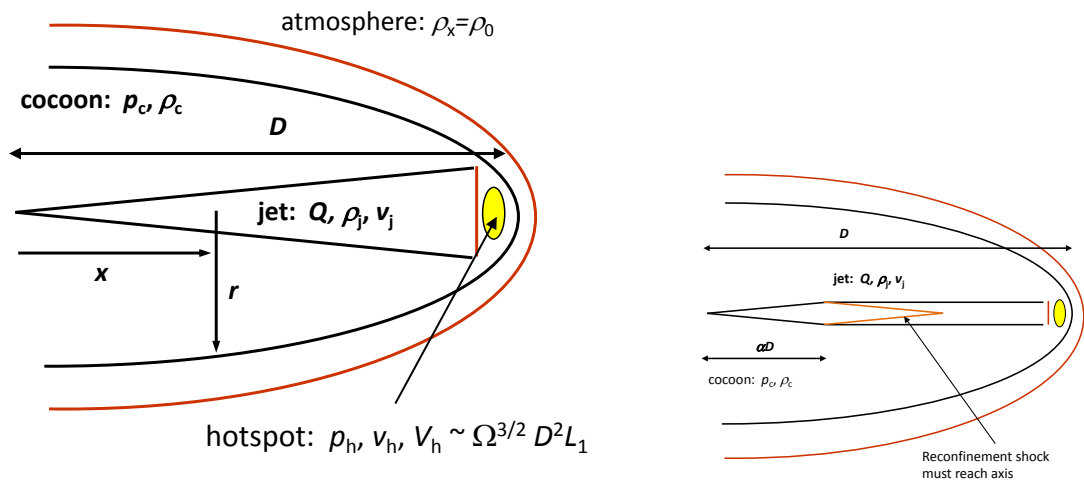

Figure 5: Figure illustrating the main components of the early-stage radio source model

Note that we also expect the solution to change it's character substantially for scales less than and greater than the characteristic length $L_{1 b}$ (see Figure 5).

I now give a brief overview of this model. Conservation of momentum at the contact surface at the head of the jet gives $\rho_{j}\left(v_{j}-\dot{D}\right)^{2} \sim \rho_{X} \dot{D}^{2} \sim p_{h}$, where the subscript $h$ refres to the hotspot. Since the jet density decreases in the uncollimated flow as $\rho_{j}=2 Q / \Omega D^{2} v_{j}^{3}$ we can solve for the jet (or source) size as a function of time giving:

$$
D=L_{1 b}\left[\left(\frac{2 v_{j} t}{L_{1 b}}+1\right)^{1 / 2}-1\right] \quad \text { and } \quad \dot{D}=\frac{v_{j}}{1+D / L_{1 b}}
$$

I now apply energy conservation to the cocoon and hotspot

$$
\frac{1}{\gamma_{c}-1} V_{c} \frac{d p_{c}}{d t}+\frac{\gamma_{c}}{\gamma_{c}-1} p_{c} \frac{d V_{c}}{d t}+\frac{1}{\gamma_{h}-1} V_{h} \frac{d p_{h}}{d t}+\frac{\gamma_{h}}{\gamma_{h}-1} p_{h} \frac{d V_{h}}{d t}=Q
$$

and take the hotspot volume to be given by $V_{h} \sim \Omega^{3 / 2} D^{2} L_{1}$. For the cocoon volume I assume the sideways expansion is highly supersonic and apply momentum conservation to give for the cocoon radius and volume

$$
r=\int_{t=t_{1}(x)}^{t}\left(\frac{p_{c}}{\rho_{X}}\right)^{1 / 2} d t \quad \text { and } \quad V_{c}=\int_{x=0}^{D} \pi r^{2}(x) d x
$$

An approximate analytical solution [?] (which is in fact accurate to a few percent for all source sizes) is given by

$$
p_{c} \propto \frac{Q}{v_{j} L_{1}^{2}}\left(\frac{D}{L_{1}}\right)^{-1}\left(1+\frac{D}{K L_{1 b}}\right)^{-1 / 2} \quad \text { and } \quad V_{c} \propto L_{1}^{3}\left(\frac{D}{L_{1}}\right)^{2}\left(1+\frac{D}{K L_{1 b}}\right)^{3 / 2}
$$

Where $K$ is a constant of order unity and the constants of proportionality are also of order unity.

This solution holds until the cocoon pressure becomes comparable with the sideways ram pressure of the jet. It is easy to show that this must always occur. The sideways ram pressure of the jet is given by

$$
\rho_{j} v_{j}^{2} \sin ^{2} \theta=\left(\frac{D}{L_{1 b}}\right)^{-2} \rho_{X} v_{j}^{2} \sin ^{2} \theta
$$




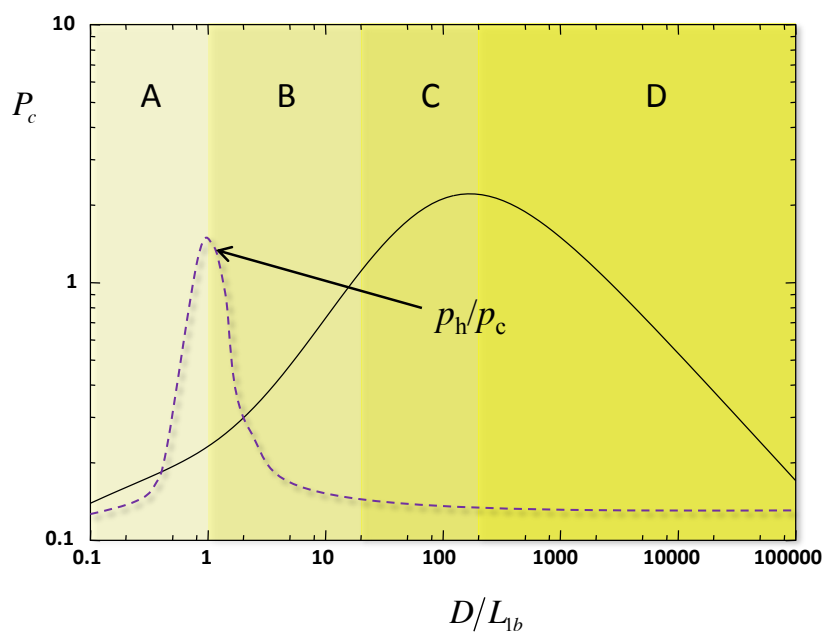

Figure 6: Figure illustrating the overall luinosity evolution for the source model described in this paper

while for large $D$ the cocoon pressure from above scales with $D$ as $D^{-3 / 2}$. Eventually the cocoon pressure must become large enough to collimate the jet and this it does by driving an oblique shock into the jet. Once the shock reaches the symmetry axis the jet is collimated and reaches pressure balance with the cocoon: this is of course precisely the situation we considered above for the self-similar evolutionary stage. This collimation transition can be solved for relatively easily.

Summarising the dynamical evolution we have therefore:

- For $D<<L_{1 b}$ the jet is overdense and propagates nearly balistially forming a small cocoon.

- The first transition occurs when $D \sim L_{1 b}$ when the jet becomes under dense and a large cocoon begins to form. It is possible to show that at this stage the ratio of hotspot to cocoon pressure reaches a maximum value.

- A second transition occurs for $D \sim 5-10 L_{1 b}$ when the jet is collimated by the cocoon pressure and we transition to the self-similar flow.

\section{Overall Evolution}

This dynamical model enables us to predict the track of a model source through the powerlinear-size (P-D) plane. A full calculation of the luminosity of the source shiuld include relevant radiative losses as well as include contributions to the luminosity from the cocoon, hotspot and jet (all being potentially orientation dependent for different phases of the source evolution. A full analysis of this form will appear elsewhere. Here I illustrate the behaviour approximately using simple equalipartition arguments mostly.

In Figure 6 the preducted cocoon luminosity is platted as a function of linear size with an indication of the hotspot to cocoon pressure ratio also shown on an arbitrary logarithmic scale. Four different phases are shown: 
A: This is the initial phase in which the jet is overdense. The luminosity of the cocoon grows slowly in this phase as $D^{1 / 4}$. The full calculation of the luminosity should include also the jet which given the conical flow behavious is expected to show the canonical flat-spectrum and may dominate the luminosity when the jet is pointing towards the observer.

B: This phase and subsequent phases have an underdense jet. Here the luminosity of the cocoon increases as $D^{7 / 8}$. At around the transition point the hotspot to cocoon pressure ration reaches a maximum and the source can appear hotspot dominated. At the end of this phase the jet becomes collimated and we enter the self-similar evolutionary period.

C: The source has now entered the self-similar evolutionary phase. Without radiative losses the cocoon (and source) luminosity would increase as $D^{2 / 3}$ in a constant external density. In fact [11] radiative losses can be significant owing to the large magnetic fields at this time and if the loss time is comparable to the dynamical time the source can enter an effective coasting phase where the luminosity is approximately constant with source size.

D: Here we are in the self-similar phase and the source is now larger than the host galaxy and I have assumed there is a declining atmosphere which causes the source luminosity to decrease as $D^{(8-7 \beta) / 12}$. Eventually radiative losses will again become significant and the source luminosity will fall faster with increasing size than indicated [12].

\section{References}

[1] S. Shabala and P. Alexander, ApJ 699 (2009) 525

[2] e.g. A.C. Fabian et al., MNRAS Lett. 344 (2003) 43

[3] e.g. D.J. Croton et al., MNRAS 365 (2006) 11

[4] e.g. E. Churazov et al., ApJ 554 (2001) 261

[5] C. Kaiser and P. Alexander MNRAS 305 (1999) 707

[6] P. Alexander MNRAS 335 (2002) 610

[7] e.g. J. Basson and P. Alexander MNRAS 339 (2003) 353

[8] C. Kaiser and P. Alexander MNRAS 286 (1997) 215

[9] N. de Vries et al., A\&A 498 (2009) 641

[10] P. Alexander MNRAS 368 (1997) 1404

[11] C. Kaiser and P.N. Best MNRAS 381 (2007) 1548

[12] P. Alexander MNRAS 319 (2000) 8 